\title{
EXTREMAL INTERPOLATORY PROBLEMS IN THE UNIT DISC
}

\section{BERNARD EPSTEIN AND JACK MINKER}

1. Introduction. In this paper we consider two extremal interpolatory problems in the unit disc, $\Delta$. We first consider the class $L^{2}(\Delta)$ consisting of all analytic functions which are quadratically integrable in $\Delta$, with norm given by

$$
\|f\|_{L}=\left(\iint_{\Delta}|f(z)|^{2} d x d y\right)^{1 / 2}<\infty .
$$

From among all functions in $L^{2}(\Delta)$ which satisfy the finite set of interpolation conditions

$$
f\left(z_{k}\right)=a_{k}, \quad k=1,2, \cdots m
$$

we determine the unique function having minimum norm (1). The (distinct) points $\left\{z_{k}\right\}$ are arbitrarily distributed over the disc, and the constants $\left\{a_{k}\right\}$ are arbitrary. In the second problem we consider the class of functions $H_{n}^{2}(\Delta)$, consisting of functions analytic in the unit disc, with norm

$$
\|f\|_{H, n}=\left(\int_{C} n(z)|f(z)|^{2}|d z|\right)^{1 / 2}<\infty,
$$

where $C$ is the unit circle $|z|=1$, and $n(z)$ is a positive continuous weight function defined on $C$. For this class we determine the unique function which satisfies the infinitely many interpolation conditions

$$
f\left(z_{k}\right)=\delta_{k j}, \quad k=1,2, \cdots
$$

and has minimum norm (3). The (distinct) points $\left\{z_{k}\right\}$ lie in $\Delta$ and $\prod_{k=1}^{\infty}\left|z_{k}\right|$ is assumed to converge. By the integral in (3) is meant $\lim _{r \rightarrow 1} \int_{C_{r}} n\left(e^{i \theta}\right)|f(z)|^{2}|d z|$, where $C_{r}$ is the circle $|z|=r, 0<r<1$.

Although the results presented here are not new, the methods are, we believe, of some interest.

2. Hilbert space concepts. In both of the above problems it is the fact that the class of functions with which we are concerned forms a Hilbert Space that is the important factor. Before proceeding with these problems it would be well to outline briefly the reasoning employed to show that a Hilbert space is formed (cf. [4]). Throughout

Received by the editors August 3, 1959 and, in revised form, November 17, 1959. 
this paper we shall refer to an arbitrary Hilbert space with norm denoted by $\|f\|$ and inner product by $(f, g)$ without subscripts whenever the discussion is applicable to both $L^{2}(\Delta)$ and $H_{n}^{2}(\Delta)$. Indeed, the ideas presented here are applicable for domains other than the disc.

That these spaces are linear and normed is trivial. Completeness follows from an inequality of the form:

$$
|f(t)| \leqq M\|f\|,
$$

where $t$ is a point of the disc and $M$ is a number depending on $t$ but not on the function $f(z)$. Such an inequality is found for the class $L^{2}(\Delta)$ by applying the mean value theorem for harmonic functions; this yields the inequality:

$$
|f(t)| \leqq \frac{1}{\pi^{1 / 2} r}\|f\|_{L},
$$

where $r$ is the distance from the point $t$ to the boundary of the disc. For the class $H_{n}^{2}(\Delta)$, we may replace $n(z)$ by unity and $f(z)$ is replaced by $f(z) N(z)$ in (3) where $N(z)$ is analytic in the disc and $|N(z)|^{2}$ $=n(z)$ almost everywhere on $C$ and solve the equivalent problem (cf. [2, p. 146]). When this is done and the Cauchy integral formula is applied to the analytic function $f^{2}(z) N^{2}(z)$ we obtain

$$
|f(t)| \leqq \frac{1}{(2 \pi r)^{1 / 2} N(t)}\|f\|_{H, n} .
$$

The inequality (5) further shows that the value of the function at a fixed point $t$ of $\Delta$ is a bounded linear functional. Hence, by the well-known theorem of Riesz and Fréchet, the linear functional $L(f)=f(t)$, can be expressed as an inner product, that is:

$$
L(f)=f(t)=(f, g) .
$$

The function $g(z)$ is a (uniquely determined) function belonging to the Hilbert space under consideration. Because of $(8), g(z)$ will be called the reproducing kernel function. For the class $L^{2}(\Delta)$ the kernel function is called the Bergman Kernel Function and will be denoted by $K_{B}(z, \bar{t})$. For the class $H_{n}^{2}(\Delta)$ the kernel function is called the Szegö Kernel Function and will be denoted by $K_{S}^{n}(z, \bar{t})$.

Given a complete orthonormal sequence for a Hilbert space admitting a kernel function, the kernel function can be obtained by forming the sum

$$
\sum_{k=1}^{\infty}\left[\phi_{k}(t)\right]-\phi_{k}(z)
$$


where the functions $\left\{\phi_{k}\right\}$ form an orthonormal basis for the space (cf. [4]). For the spaces $L^{2}(\Delta)$ and $H_{n}^{2}(\Delta)$, the functions $\left\{z^{k}\right\}, k=0,1,2, \cdots$ form a complete orthogonal system for both $L^{2}(\Delta)$ and $H_{n}^{2}(\Delta)$. By normalizing this sequence of functions for each of the spaces $L^{2}(\Delta)$ and $H_{n}^{2}(\Delta)$, and applying (9), we get

$$
\begin{aligned}
& K_{B}(z, \bar{t})=\frac{1}{\pi} \frac{1}{(1-z \bar{t})^{2}}, \\
& K_{S}^{n}(z, \bar{t})=\frac{1}{2 \pi} \frac{1}{1-\bar{t}_{z}} \frac{1}{N(z)[N(t)]^{-}} .
\end{aligned}
$$

3. The projection operator. Returning to the extremal problems posed in $\$ 1$, it is readily seen that if there exists one function of the space under consideration which interpolates correctly at the prescribed points, then both the existence and uniqueness of a solution to the proposed extremal problem are assured. It is further seen that the extremal function is uniquely characterized by its interpolation property and the fact that it is orthogonal to the subspace of functions $Z$ which vanish at all the points of interpolation. This condition may be written as follows:

$$
P f=0,
$$

where $f$ is the extremal function and $P$ denotes the orthogonal projection operator onto $Z$. Thus, if $w(z)$ is any "competing" function, the extremal function is given by

$$
f(z)=w(z)-P w(z),
$$

and the minimum norm is given by

$$
(f, f)=(w, w-P w) .
$$

(It is obvious in the case of a finite set of interpolation conditions that "competing" functions exist.)

Let $P_{m}$ be the projection operator associated with $m$ points of interpolation. (In the case where there are infinitely many points of interpolation we shall consider the first $m$ points and then let $m$ tend to infinity.) Then, for both of the aforementioned Hilbert spaces, the operator $P_{m}$ will be constructed with the aid of the bounded linear operator $T_{m}$, where

$$
T_{m} g=B_{m}(z) g(z),
$$

$g(z)$ being in $L^{2}(\Delta)$ or $H_{n}^{2}(\Delta)$ and $B_{m}(z)$ is the Blaschke product: 


$$
B_{m}(z)=\prod_{k=1}^{m} \frac{\left[z_{k}\right]^{-}}{\left|z_{k}\right|} \frac{z-z_{k}}{1-\left[z_{k}\right]^{-z}} .
$$

The points $\left\{z_{k}\right\}$ in (15) are the points of interpolation.

If $T_{m}^{*}$ denotes the adjoint of $T_{m}$ and if the (bounded) operator $\left(T_{m}^{*} T_{m}\right)^{-1}$ exists (which will be shown shortly), then it is easily seen that the projection operator may be represented as follows:

$$
P_{m}=T_{m}\left(T_{m}^{*} T_{m}\right)^{-1} T_{m}^{*} .
$$

Using the same reasoning as in [1], the adjoint operator may be written as

$$
T_{m}^{*} g(t)=\left(\left[B_{m}(z)\right]-g(z), K(z, \grave{t})\right),
$$

where $K(z, t)$ is the reproducing kernel function.

\section{Solution of the extremal problems.}

A. The extremal problem for $L^{2}(\Delta)$. We first consider the extremal problem posed for the functions of class $L^{2}(\Delta)$. We temporarily assume that none of the points $z_{k}$ is the origin. Let the operator $T_{m}$ be defined by (14), where $g(z) \in L^{2}(\Delta)$. The operator $T_{m}$ maps all functions in $L^{2}(\Delta)$ into the class of functions $Z \subset L^{2}(\Delta)$. Now, given any function $h(z) \in L^{2}(\Delta)$, the function $h(z) / B_{m}(z) \in L^{2}(\Delta)$, thereby providing a factorization. That this is so can be seen by drawing a disc, $\Delta_{k}$, of radius $r$ about each of the points of interpolation (i.e., the zeros of $B_{m}(z)$ ), where none of the discs intersect, and each disc is contained within $\Delta$. We may therefore write:

$$
\begin{aligned}
& \iint_{\Delta}\left|\frac{h(z)}{B_{m}(z)}\right|^{2} d x d y \\
& \quad \leqq c_{1} \sum_{k=1}^{m} \iint_{\Delta_{k}}\left|\frac{h(z)}{z-z_{k}}\right|^{2} d x d y+c_{2} \iint_{\Delta-\sum_{k=1}^{m} \Delta_{k}}|h(z)|^{2} d x d y,
\end{aligned}
$$

where

$$
c_{1}=\max _{k=1, \ldots, m}\left\{\sup _{z \in \Delta_{k}}\left|\frac{z-z_{k}}{B_{m}(z)}\right|^{2}\right\}
$$

and

$$
c_{2}=\sup _{z \in \Delta-\sum_{k=1}^{m} \Delta_{k}}\left(\left|B_{m}(z)\right|^{-2}\right) .
$$


Furthermore, by expanding $h(z)$ in a Taylor series and using the orthogonality of the functions $\left\{\left(z-z_{k}\right)^{j}\right\}$ over the disc $\Delta_{k}$, it is readily seen that

$$
\iint_{\Delta_{k}}\left|\frac{h(z)}{z-z_{k}}\right|^{2} d x d y \leqq \frac{2}{r^{2}} \iint_{\Delta}|h(z)|^{2} d x d y .
$$

It then follows that

$$
\iint_{\Delta}\left|\frac{h(z)}{B_{m}(z)}\right|^{2} d x d y \leqq c \iint_{\Delta}|h(z)|^{2} d x d y,
$$

where $c$ is a constant. From (14) it is obvious that $\left\|T_{m}\right\|_{L}$ $\leqq \sup _{z \in \Delta}\left|B_{m}(z)\right|$, which with (20) yields

$$
0<\frac{1}{c} \leqq \frac{\left(T_{m} g, T_{m} g\right)}{(g, g)} \leqq\left\|T_{m}\right\|^{2}<\infty, \quad g \in L^{2}(\Delta) .
$$

Conditions (21), however, assure the existence of the bounded operator $\left(T_{m}^{*} T_{m}\right)^{-1}$ (cf. [5, p. 263]).

The operator $T_{m}^{*} T_{m}$ is then given by:

$$
T_{m}^{*} T_{m} g(z)=\iint_{\Delta}\left(B_{m}(\zeta) g(\zeta)\right)\left[B_{m}(\zeta) \frac{1}{\pi} \frac{1}{(1-\bar{z} \zeta)^{2}}\right]^{-} d \xi d \eta
$$

or

$$
T_{m}^{*} T_{m} g(z)=\frac{1}{2 i} \int_{C} B_{m}(\zeta) g(\zeta)\left[\int^{\zeta} B_{m}(u) \frac{1}{\pi} \frac{1}{(1-\bar{z} u)^{2}} d u\right]^{-} d \zeta .
$$

(22a) is obtained by combining (10a), (14) and (17). Applying Green's formula, we derive (22b). Expanding $B_{m}(u)\left(1 /(1-\bar{z} u)^{2}\right)$ in its partial fractions and employing the formula

$$
\frac{1}{2 \pi i} \int_{G} g(u)[\log (1-\bar{z} u)]-d u=-\int_{0}^{z} g(t) d t, \quad z \in \Delta,
$$

(22b) becomes:

$$
T_{m}^{*} T_{m} g(z)=\frac{d}{d z}\left\{\frac{1}{B_{m}(z)} \int_{0}^{z} B_{m}(u) g(u) d u\right\}+\sum_{k=1}^{m} \frac{\int_{0}^{z_{k}} B_{m}(u) g(u) d u}{B_{m}^{\prime}\left(z_{k}\right)\left(z_{k}-z\right)^{2}}
$$

which gives an explicit expression for the operator $T_{m}^{*} T_{m}$.

If we now let $u(z)=T_{m}^{*} T_{m} g(z)$ and solve for $g(z)$ in terms of $u(z)$ we get $g(z)=\left(T_{m}^{*} T_{m}\right)^{-1} u(z)$. Therefore, if we substitute $u(z)$ for $T_{m}^{*} T_{m} g(z)$ in (23) and solve the resulting integral equation for $g(z)$ in terms of 
$u(z)$, we find the inverse operator for $T_{m}^{*} T_{m}$. Performing this substitution, we observe that, as the equation stands, we cannot solve for $g(z)$ in terms of $u(z)$, since the constants $\int_{0}^{z_{k}} B_{m}(u) g(u) d u$ depend upon $g(z)$. If, however, we integrate this equation between the limits 0 and $z_{j}$ we get:

$$
\begin{aligned}
\int_{0}^{z_{j}} u(z) d z= & \sum_{k=1 ; k \neq j}^{m} \frac{\int_{0}^{z_{k}} B_{m}(\rho) g(\rho) d \rho}{B_{m}^{\prime}\left(z_{k}\right) z_{k}\left(z_{k}-z_{j}\right)} z_{j} \\
& -\left[\frac{B_{m}^{\prime \prime}\left(z_{j}\right)}{2\left(B_{m}^{\prime}\left(z_{j}\right)\right)^{2}}+\frac{1}{z_{j} B_{m}^{\prime}\left(z_{j}\right)}\right] \int_{0}^{z_{j}} B_{m}(\rho) g(\rho) d \rho .
\end{aligned}
$$

Equations (24) (with $j=1, \cdots, m$ ) constitute a linear system which we can solve for each $\int_{0}^{z_{k}} B_{m}(\rho) g(\rho) d \rho$ as a linear combination of the quantities $\int_{0}^{z_{i}} u(z) d z$. If the determinant of the system (24) were zero, it would be possible to choose constants $a_{k}$ so that the system (24) is unsolvable while we know the system is always solvable. Hence the determinant of (24) does not vanish, therefore, we may write

$$
\sum_{j=1}^{m} b_{k_{j}} \int_{0}^{z_{k}} u(z) d z=\int_{0}^{z_{k}} B_{m}(\rho) g(\rho) d \rho,
$$

where the $b_{k j}$ are constants determined by the points $z_{1}, z_{2}, \cdots, z_{m}$.

Substituting (25) into (23) and solving the resulting integral equations we find

$$
\begin{aligned}
& \left(T_{m}^{*} T_{m}\right)^{-1} g(z) \\
& \quad=\frac{1}{B_{m}(z)} \frac{d}{d z}\left\{B_{m}(z) \int_{0}^{z}\left[g(z)-\sum_{k, j=1}^{m} \frac{b_{k j} \int_{0}^{z k} g(t) d t}{B_{m}^{\prime}\left(z_{j}\right)\left(z_{j}-z\right)^{2}}\right] d z\right\} .
\end{aligned}
$$

Applying (14), (17) and (26) to (12) we find that the solution to the first extremal problem is given by:

$$
f(z)=\frac{d}{d z}\left\{B_{m}(z) \sum_{j, k=1}^{m} \frac{b_{k j} f\left(z_{k}\right)}{B_{m}\left(z_{j}\right) B_{m}^{\prime}\left(z_{k}\right)} \cdot \frac{z}{z_{j}\left(z_{j}-z\right)}\right\} .
$$

The norm of the extremal function is readily calculated by an application of the method of residues to (22b) if we take advantage of the fact that on $C$ we have $\bar{z}=1 / z$ and $\left[B_{m}(z)\right]^{-}=1 / B_{m}(z)$.

In case the origin is one of the $m$ points of interpolation we define a new bounded linear operator $T_{m}^{\prime} g=z B_{m-1}(z) g(z)$ and proceed in exactly the same manner as before. 
B. Extremal problems for $H_{n}^{2}(\Delta)$. We first consider the case of a finite set of points (including perhaps the origin) arbitrarily distributed in the unit disc and prescribe the interpolatory values $\delta_{i j}$ where $j$ is given, $1 \leqq j \leqq m$. Again we consider the operator (14), where $g(z)$ is now in $H_{n}^{2}(\Delta)$. Then,

$$
\begin{aligned}
\left\|T_{m} g\right\|_{H, n}^{2} & =\int_{C}\left|N(z) B_{m}(z) g(z)\right|^{2}|d z|=\int_{C}|N(z) g(z)|^{2}|d z| \\
& =\|g\|_{k, n}^{2},
\end{aligned}
$$

which follows since $\left|B_{m}(z)\right|=1$ on $C$. As before, this assures that the operator $T_{m}^{*} T_{m}$ can be inverted. Indeed, by applying (17) and exploiting the reproducing property of the Szegö kernel function $K_{S}^{n}(z, \bar{t})$, cf. (10b), we readily find that $T_{m}^{*} T_{m}$ is the identity operator and thus is its own inverse. Thus, it follows from (16) that the projection operator $P_{m}$ is given by $P_{m} g=T_{m} T_{m}^{*} g$.

Evaluating $P_{m} u$ for the function

$$
u(z)=\frac{1}{B_{m}^{\prime}\left(z_{j}\right)} \frac{B_{m}(z)}{\left(z-z_{j}\right)},
$$

(where we note that $u(z)$ satisfies $u\left(z_{i}\right)=\delta_{i j}$ and $u(z)=H_{n}^{2}(\Delta)$ ) we find:

$$
\begin{aligned}
P_{m} u & =T_{m} T_{m}^{*} u \\
& =B_{m}(z) \int_{C}|N(t)|^{2}\left[B_{m}(t)\right]^{-} \frac{K_{S}(z, \bar{t})}{N(z)[N(t)]^{-}} \frac{B_{m}(t)}{B_{m}^{\prime}\left(z_{j}\right)\left(t-z_{j}\right)}|d t| .
\end{aligned}
$$

Substituting (10b) into (30) and noting that on $C$ we have $\left|B_{m}(t)\right|^{2}=1$ and $|d t|=d t / i t$ we evaluate (30) by the method of residues, obtaining

$$
P_{m} u=\frac{B_{m}(z)\left[N\left(z_{j}\right)-N(z)\right]}{N(z) B_{m}^{\prime}\left(z_{j}\right)\left(z_{j}-z\right)}
$$

The extremal solution, obtained from (12) and (30), is then given by

$$
f_{m, j}(z)=\frac{B_{m}(z) N\left(z_{j}\right)}{\left(z-z_{j}\right) B_{m}^{\prime}\left(z_{j}\right) N(z)} .
$$

Now, given a countable set of points $\left\{z_{k}\right\}$ in $\Delta$ for which $\prod_{k=1}^{\infty}\left|z_{k}\right|$ converges, the corresponding infinite Blaschke product converges in the mean square on the circumference. Within the disc the convergence is uniform on any closed set, and the convergence is to a function of modulus not greater than unity in the disc (cf. [2, p. 283]). 
Thus, the sequence of functions (32) converges uniformly everywhere in $\Delta$ to some analytic function. This function obviously does not vanish identically and is the solution to the extremal problem with interpolatory conditions (4).

Turning again to the finite interpolation problem where the values $\left\{a_{j}\right\}$ are arbitrarily prescribed at the points $\left\{z_{j}\right\}$, we remark that the extremal solution is given by

$$
g_{m}(z)=\sum_{j=1}^{m} a_{j} f_{m, j}(z)
$$

where $f_{m, j}(z)$ is defined by (32). This follows from the fact that the extremal solution is characterized uniquely among all "competing" functions by the property that it is orthogonal to all functions in $H_{n}^{2}(\Delta)$ which vanish at the points $\left\{z_{j}\right\}$. The norm is then easily calculated by the method of residues.

\section{BIBLIOGRAPHY}

1. B. Epstein, D. S. Greenstein, and J. Minker, An extremal problem with infinitely many interpolatıon conditions, Ann. Acad. Sci. Fenn. Ser. A-I-250/10, 1958, pp. 1-10.

2. J. L. Walsh, Interpolation and approximation by rational functions in the complex domain, Amer. Math. Soc. Colloquium Publications, no. 20, 2d ed., 1956.

3. S. Takenaka, On orthogonal functions and a new formula of interpolation, Jap. J. Math. vol. 2 (1925) pp. 129-145.

4. S. Bergman, The kernel function and conformal mapping, Mathematical Surveys, no. 5, Amer. Math. Soc. 1950.

5. F. Riesz and B. Sz.-Nagy, Functional analysis (translated from the $2 \mathrm{~d}$ French ed. by Leo F. Boron), New York, Frederick Ungar Publishing Co., 1955.

University of Pennsyluania and

Radio Corporation OF AMERICA 